\title{
Correlation of Antepartum Uterine Activity and Cervical Change in Twin Gestation
}

\author{
R. B. Newman, J. M. Ellings, M. M. O'Reilly, B. C. Brost, M. C. Mil- \\ ler III, D. Gates Jr \\ Department of Obstetrics and Gynecology; Biostatistics, Epidemiology and Statistical \\ Sciences, Medical University of South Carolina, Charleston, South Carolina
}

Objective. To determine the correlation between quantitative measurements of antepartum uterine activity and cervical change twin gestations.

Methods. Forty women from our Twin Clinic constituted the study group. Participants had a cervical examination each week between 20 and 37 weeks gestation and a cervical score (CS) was calculated as follows: CS = cervical length (cms)-cervical dilation (cms) at the internal os. The women also performed blinded home uterine activity monitoring (HUAM) for a mean of $7.0+3.0 \mathrm{hrs} / \mathrm{wk}(+\mathrm{SD})$. Uterine activity was expressed as mean number of contractions/hour/week gestation based on the average of three independent reviewers. CS was determined by a single clinician unaware of the HUAM recordings. A significant change in the CS was defined as a reduction of at least 0.5 from the preceding week. Correlation coefficients were used to determine the association between uterine activity and change in the cervical score.

Results. Twin pregnancy was characterized by a rise from $0.2+.03$ contractions/hr at 20 weeks to $3.2+2.4$ contractions $/ \mathrm{hr}$ at 37 weeks gestation. CS fell from a mean of $2.6+0.2$ at 20 weeks to $-2.1+0.9$ at 37 weeks gestation. There was a significant negative correlation $(-0.317, \mathrm{p}<.0001)$ between increasing uterine activity and decreasing CS. There were significantly more $(\mathrm{p}<.002)$ contractions during the 7 days preceding a significant reduction in CS $(3.3+3.5$ contractions/hr) than when the CS was unchanged $(1.6 \pm 1.5$ contractions/hr).

Conclusions. In twin gestations, an increasing frequency of uterine contractions is strongly correlated with quantifiable cervical change between 20-37 weeks gestation. Persistent daytime contraction frequencies of $>3 / \mathrm{hr}$ represent a risk factor for cervical dilation and/or effacement.

Key words: Uterine contractions, Cervical score, Cervical change, Twin gestations, Uterine activity monitoring, Preterm labor 


\section{INTRODUCTION}

The frequency of antepartum uterine contractions $[1,2,3]$ and cervical status $[4,5]$ have both been investigated as factors associated with preterm delivery in twin gestations. While the progressive change in prelabor cervical status would appear to mirror the crescendo of uterine activity with advancing gestation, these two parameters have never been simultaneously studied. An understanding of this relationship is obviously important to our appreciation of the causes of preterm labor in twin gestations. The purpose of this study was to determine the correlation between quantitative measurements of antepartum uterine activity and simultaneous cervical change in twin gestations. If such a correlation does exist, then a second objective will be to determine the mean contraction frequency associated with cervical change assessed on a week to week basis.

\section{MATERIALS AND METHODS}

Forty women from a specialized Twin Clinic at the Medical University of South Carolina constituted the study group. The study protocol was approved by the Medical University of South Carolina Institutional Review Board. All participants were 18 years of age or older, had uncomplicated twin gestations, were between 20 and 37 weeks gestation, and had access to a touchtone telephone. Study personnel interviewed all eligible patients to determine their willingness to participate. Approximately $70 \%$ of eligible patients consented to participate in the study. Only $4(10 \%)$ patients failed to adequately comply with study requirements.

Patients were instructed in the use of a lightweight tocodynamometer designed for outpatient use (Tokos Medical Corporation; Santa Ana, California) [6]. Patients monitored uterine activity one to two hours each day. Monitoring was performed in either the morning or the afternoon and was required at least five days per week to remain in compliance with the study protocol. Neither the study participants nor the clinicians were aware of the Home Uterine Activity Monitoring (HUAM) recordings. The research nurse receiving the HUAM telephone transmissions provided no feedback to the patients and stored the data by code number for evaluation at study completion. HUAM was continued until one of several study endpoints occurred. These endpoints included attainment of 37 weeks gestation [11], the occurrence of preterm labor [10], preterm premature rupture of the membranes [8], or preterm delivery for maternal or fetal indications [7]. As previously mentioned, four other women failed to comply with HUAM requirements and were ultimately dropped from study participation.

Study patients received weekly prenatal visits through a specialized twin clinic. Care providers included a single attending obstetrician specializing in maternal-fetal medicine and a certified nurse midwife who performed all cervical examinations. At each weekly visit, a digital examination was done to evaluate cervical dilation at the internal os and cervical length. A cervical score was calculated as described by Houlton et al [7]. In brief, cervical score is determined as follows: cervical length (in centimeters) minus cervical dilation at the internal os (in centimeters). For example, a cervix that is 2 centimeters long with a closed internal os gives a score of +2 . A cervix that is 1 centimeter long with the cervix dilated 1 centimeter at the internal os gives a score of zero. A cervix that 
is 1 centimeter long with the internal os dilated 3 centimeters gives a score of -2 . All cervical scores were calculated by a single clinician unaware of the HUAM data. No prophylactic tocolysis or cerclages were used.

Preterm labor was diagnosed based on the criteria of Gonik and Creasy [3]; the presence of regular uterine contractions ( 4 in 20 minutes or 8 in 60 minutes) between 20 and 37 completed weeks accompanied by either documented cervical change, initial cervical dilation $\geq 2 \mathrm{~cm}$, initial cervical effacement of $\geq 80 \%$, or preterm premature rupture of the membranes (pPROM). Preterm birth was defined as delivery before 37-0/7 completed weeks of gestation. Gestational age was based on the estimated date of confinement predicted by the first day of the last menstrual period if confirmed by ultrasonography. If there was a discrepancy of $>$ one week in the first trimester, $>$ ten days in the second trimester, or $>$ three weeks in the third trimester between menstrual and ultrasonic dating criteria, then the estimated date of confinement was recalculated based on ultrasonographic findings.

A mean uterine contraction frequency per hour per gestational week was calculated. Uterine contractions were defined as $>5$ millimeters amplitude and $>35$ seconds duration with a gradual upslope and downslope typical of a uterine contraction. The number of uterine contractions on each HUAM recording was determined based on the average of 3 independent and blinded reviews of each uterine monitoring strip. That number was divided by the number of hours of monitoring for each gestational week to determine the mean contraction frequency/hour per gestational week for each patient. The cervical score was calculated at the end of each week of uterine activity monitoring. A significant change in the cervical score was any reduction of at least 0.5 from the preceding week. Results are expressed as means \pm standard deviation. A correlation coefficient was calculated to determine the relationship between contraction frequency and a change in cervical score. Individual comparisons of contraction frequency or cervical score employed the Student's t-test with significance established at $\mathrm{p}<0.05$.

\section{RESULTS}

The forty study participants had a mean maternal age of $24.9 \pm 5.3$ years and $15(37 \%)$ were nulliparous. The study group was primarily black $(73 \%)$, single $(60 \%)$, and publicly funded (83\%). Study participants performed HUAM $7.0 \pm 3.0 \mathrm{hrs} / \mathrm{wk}$ between $24.7 \pm 2.8$ and $33.3+3.5$ weeks gestation. Eighteen women delivered at $34.4 \pm 1.4$ weeks due to either spontaneous preterm labor (10) or pPROM (8). Fifteen of the remaining women delivered spontaneously at term $(37.8 \pm 1.5$ wks gestation $)$ while seven delivered near term $(35.0 \pm 1.2$ wks gestation) for maternal/fetal indications.

Uterine activity was characterized by a rise from $0.2 \pm .03$ contractions $/ \mathrm{hr}$ at 20 weeks to $3.2 \pm 2.4$ contractions $/ \mathrm{hr}$ at 37 weeks gestation (Figure 1). Cervical score fell from a mean of $+2.6 \pm 0.2$ at 20 weeks to $-2.1 \pm 0.9$ at 37 weeks gestation (Figure 2). There was a significant negative correlation $(\mathrm{p}<.0001)$ between increasing uterine activity and a decreasing cervical score (correlation coefficient $-0.317 ; \mathrm{R}^{2}=10.1 \%$ ).

There were significantly more $(\mathrm{p}<.002)$ contractions during the seven days preceding a significant reduction in cervical score $(3.3 \pm 3.5$ contractions $/ \mathrm{hr})$ than when the cervical score was unchanged $(1.6 \pm 1.5$ contractions $/ \mathrm{hr})$. Cumulatively, there was a small 


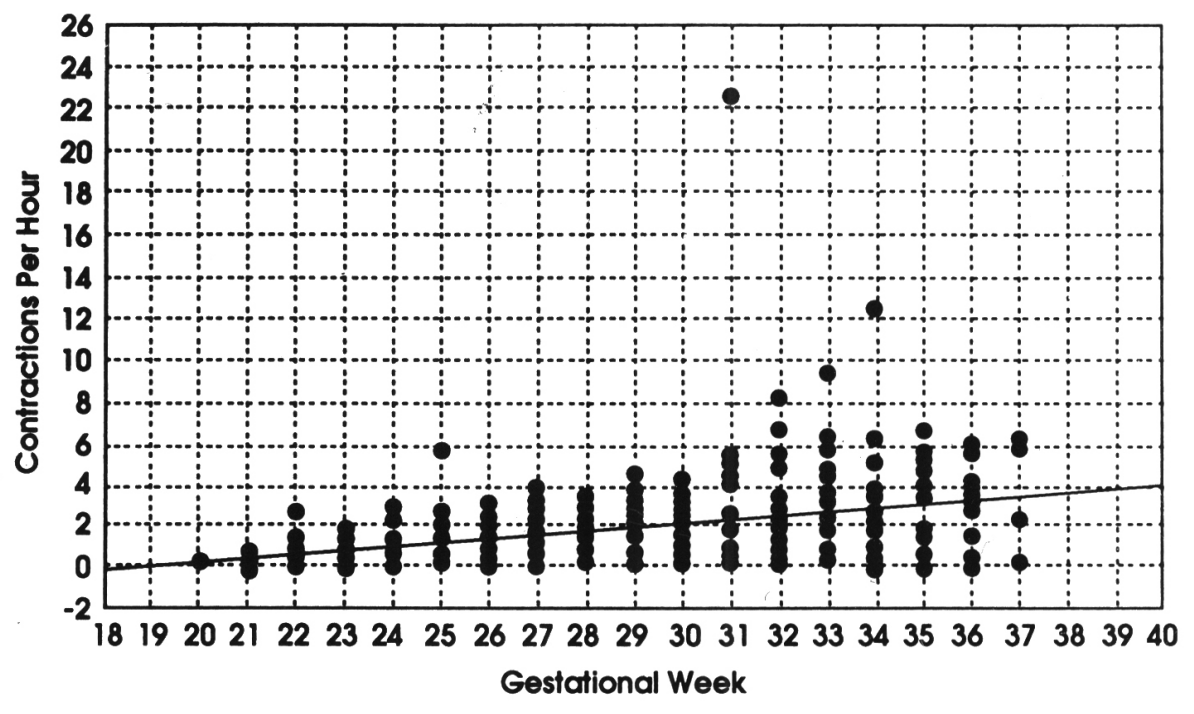

Fig. 1 - Average number of contractions per hour for twin gestations $(N=40)$ between 20 and 37 weeks gestation. Each dot represents the mean contraction frequency per hour for an individual patient that gestational week. The best fit linear regression curve is superimposed.

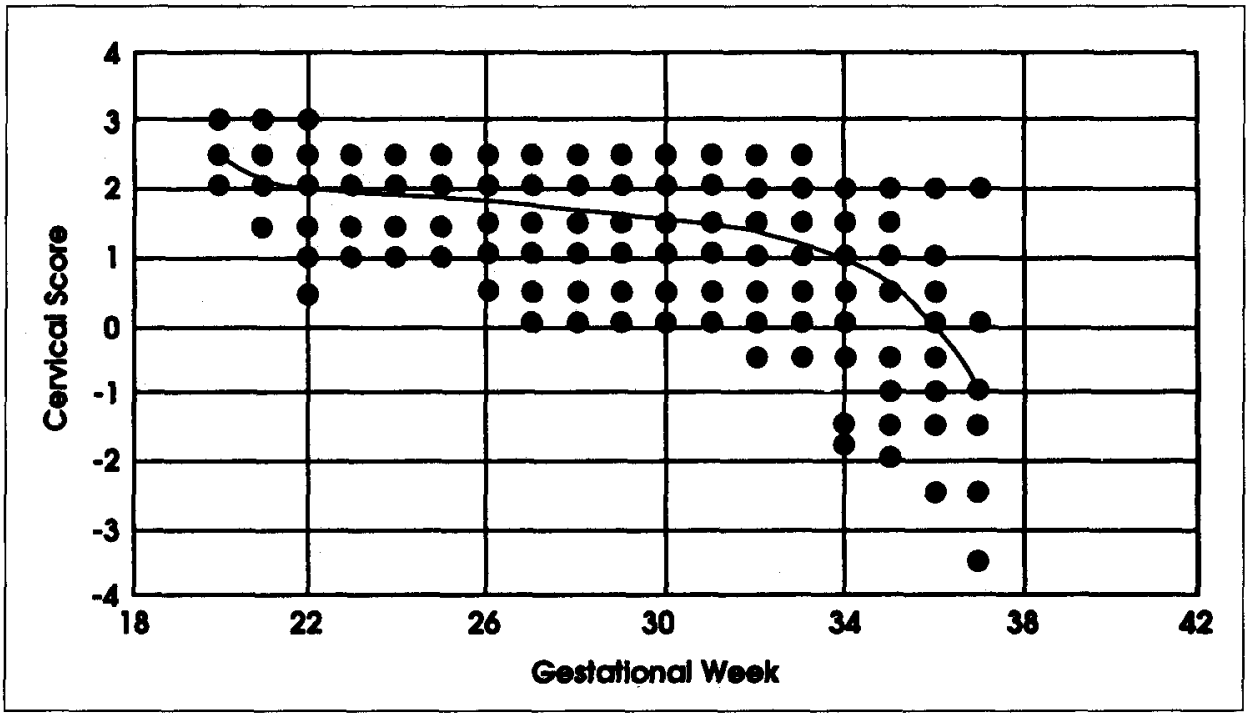

Fig. 2 - Falling cervical score in twin gestations $(N=40)$ between 20 and 37 weeks gestation. Each dot represents the cervical score for an individual patient that gestational week. The best fit polynomial regression curve superimposed. 
but significant difference $(\mathrm{p}<.0001)$ in mean cervical score between parous $(1.4 \pm 0.9)$ and nulliparous $(1.7 \pm 0.6)$ women over the entire study period. However, the differences in cervical score between parous and nulliparous women were not significant when compared on a week by week basis.

\section{DISCUSSION}

The increased frequency of prelabor uterine contractions in twin gestations has been previously reported $[1,2,3]$. These studies have demonstrated a crescendo of uterine activity preceding both term and preterm delivery in multifetal gestations. Other investigators have looked at the cervical changes preceding labor. The potential predictive value for labor of a calculated cervical score in twin gestations has been specifically studied $[4,5]$. As the cervical score falls, the mean time until delivery shortens suggesting a relationship between the state of the cervix and the timing of delivery. A cervical score of $\leq 0$ on or before 34 weeks gestation was associated with a significantly increased relative risk of preterm delivery [5]. Conversely, a cervical score of $>0$ was rarely associated with spontaneous delivery in the ensuing seven days $[4,5]$.

Although it has been generally assumed that prelabor cervical change is related to antepartum uterine activity, this relationship has never actually been simultaneously evaluated. The temporal relationship between the crescendo of prelabor uterine activity and the progressive fall in cervical score certainly suggests such a relationship. Indirect support for the relationship between prelabor uterine activity and cervical change comes from the randomized clinical trials of HUAM. Both singletons and twins using HUAM present with less cervical dilation compared to women practicing traditional preterm labor surveillance $[9,10,11,12]$. However, the variability in contraction frequency between twins who deliver prematurely and those who deliver at term has limited the ability of HUAM to anticipate preterm delivery risk.

The current study confirms that a significant relationship does exist between mean weekly uterine contraction frequency and prelabor cervical change in twin gestations. The data indicates that a fall in cervical score is preceded by an increased mean contraction frequency ( $>3$ contractions/hr) during the week preceding the reduction in cervical score.

Important considerations are the timing and interpretation of the HUAM data. Uterine activity is influenced by the time of day with higher contraction frequencies reported to occur after dark and peaking in the early nocturnal hours ( 3 to 8 a.m.) $[13,14]$. The HUAM in this study was performed either in the morning or afternoon at patient's discretion. Previous studies have not identified any significant differences in reported contraction frequencies during these daytime hours. However, these daytime hours do represent a period of relative uterine quiescence [13, 14]. Selection of these times for patient monitoring may, therefore, underestimate a given patient's overall uterine activity but should not influence our comparison of uterine activity from one monitoring session to the next.

Determination of contraction frequency can also be difficult as most of these prelabor contractions are of low pressure and amplitude. The definition of a uterine contraction used in this study is similar to that used in many previous investigations of HUAM 
data $[1,6,9,10]$. Significant inter - and intra - observer variability exists in the identification of prelabor uterine contractions, however, reliability increases significantly when three reviewers are used as in the current study protocol [15]. The reliability achieved with three reviewers is similar to that reported with computer software programs devised specifically to identify similarly defined prelabor uterine contractions [13].

This study possibly provides some insight into the etiology of preterm labor in twin gestations. An increased frequency of uterine contractions appear to be responsible in part for the increased risk of preterm delivery seen in multiple gestations. However, it is important to point out that antepartum uterine activity explained only $10 \%$ of the variability in cervical score over time. Even daily HUAM reflects only a portion of the total uterine activity to which the cervix is exposed. A greater proportion of the variability would likely have been explained if continuous uterine activity data were available. Monitoring of uterine activity in the late evening or early morning hours may have been more revealing due to the higher contraction frequencies reported at these times $[13,14]$. Unfortunately, this data is not available and a great deal of the variability in cervical status remains unexplained.

Other potential influences on the variability in cervical status include maternal parity and endocrinologic factors. Maturational influences of steroid hormones, relaxin, or prostaglandins may all affect the compliance and/or distensibility of the cervix $[16,17$, 18]. However, they do not necessarily result in cervical dilation or effacement. It is believed that these latter two processes result from the contractile activity of the uterus which gradually pulls cervical tissue upward and over the presenting part, incorporating this tissue into the isthmus of the uterus [19]. Increasing uterine activity and cervical maturation, in all likelihood, act in concert to dilate and efface the cervix in preparation for both term and preterm labor.

\section{CONCLUSIONS}

In twin gestations, an increasing frequency of uterine contractions is strongly correlated with quantifiable measures of cervical change between 20-37 weeks of gestation. Persistent daytime contraction frequencies of $>3$ /hour was associated with prelabor cervical dilation and effacement. This is the first study that simultaneously quantitates both uterine ativity data and cervical status. The study demonstrates a correlative relationship between the two supporting the belief that increased antepartum uterine activity is a major cause of preterm labor in twin gestations.

Acknowledgement: Partial financial support for this project was provided by Tokos Medical Corporation (Santa Ana, California). 


\section{REFERENCES}

1. Dyson DC, Crites YM, Ray DA, Armstrong MA (1991): Prevention of preterm birth in highrisk patients: The role of education and provider contact versus home uterine monitoring. Am J Obstet Gynecol 164: 756-62.

2. Garite TJ, Bentley DL, Hamer CA, Porto ML (1990): Uterine activity characteristics in multiple gestations. Obstet Gynecol 76: 56S-59S.

3. Germain AM, Valenzuela GJ, Ivankovic M, Ducsay CA, Gabella C, Seron-Ferre M (1993): Relationship of circadian rhythms of uterine activity with term and preterm delivery. Am J Obstet Gynecol 168: 1271-7.

4. Gonik B, Creasy RK (1986): Preterm labor: Its diagnosis and management. Am J Obstet Gynecol 154: 3-8.

5. Houlton MCC, Marivate M, Philpott RH (1982): Factors associated with preterm labour and changes in the cervix before labour in twin pregnancy. Br J Obstet Gynaecol 89: 190-4.

6. Huszar G (1981): Biology and biochemistry of myometrial contractility and cervical maturation. Semin Perinat 5: 216-35.

7. Katz M, Gill PJ (1985): Initial evaluation of an ambulatory system for home monitoring and the transmission of uterine activity data. Obstet Gynecol 66: 273-77.

8. Knupple RA, Lake MF, Watson DL, Welch RA, Hill WC, Fleming AD, Martin RW, Bentley DL, Moenning RK, Morrison JC (1990): Preventing preterm birth in twin gestation. Home uterine activity monitoring and perinatal nursing support. Obstet Gynecol 76: 24S-29S.

9. Liggins GC (1978): Ripening of the cervix. Semin Perinat 2: 261-70.

10. Moore TR, Iams JD, Creasy RK, Burau KD, Davidson AL, and the Uterine Activity in Pregnancy Working Group (1994): Diurnal and gestational patterns of uterine activity in normal human pregnancy. Obstet Gynecol 83: 517-23.

11. Morrison JC, Martin JN, Martin RW, Gookin KS, Wiser WL (1987): Prevention of preterm birth by ambulatory assessment of uterine activity: A randomized study. Am J Obstet Gynecol 156: 536-43.

12. Mou SM, Sunderji SG, Gall S, How H, Patel V, Grey M, Kayne HL, Corwin M (1991): Multicenter randomized clinical trial of home uterine activity monitoring for detection of preterm labor. Am J Obstet Gynecol 165: 856-66.

13. Neilson JP, Verkuyl DAA, Crowther CA, Bannerman C (1988): Preterm labor in twin pregnancies: Prediction by cervical assessment. Obstet Gynecol 72: 719-23.

14. Newman RB, Gill PJ, Campion S, Katz M (1989): The influence of fetal number on antepartum uterine activity. Obstet Gynecol 73: 695-9.

15. Newman RB, Gill PJ, Kate M (1986): Uterine activity during pregnancy in ambulatory patients: Comparison of singleton and twin gestations. Am J Obstet Gynecol 154: 530-31.

16. Newman RB, Godsey RK, Ellings JM, Campbell BA, Eller DP, Miller MC (1991): Quantification of cervical change: Relationship to preterm delivery in the multifetal gestation. Am J Obstet Gynecol 165: 264-71.

17. Scheerer LJ, Campion S, Katz M (1990): Ambulatory tocodynamometry data interpretation: Evaluating variability and reliability. Obstet Gynecol 76: 67S-70S.

18. Steinetz BG, O'Byrne EM, Kroc RL (1980): The role of relaxin in cervical softening during pregnancy in mammals. In Naflolin F, Stubblefield PG (eds): Dilation of the Uterine Cervix. Connective Tissue, Biology and Clinical Management". New York: Raven, pp 157-77.

19. Uldjerg N, Ekman G, Malmstrom A, Ulmsten U (1981): Biochemical and morphological changes in the human cervix after local application of prostaglandin E2 in pregnancy. Lancet 1: $267-8$

Correspondence: Roger B. Newman M. D., Department of Obstetrics and Gynecology, Medical University of South Carolina, 171 Ashley Avenue, Charleston, South Carolina 29425-2233, USA. 